\title{
Spectral Evidence of Squeezing of a Weakly Damped Driven Nanomechanical Mode
}

\author{
J. S. Huber $\odot,{ }^{1}$ G. Rastelli $\odot,{ }^{1}$ M. J. Seitner, ${ }^{1}$ J. Kölbl $\odot,{ }^{1, *}$ W. Belzig $\odot,{ }^{1}$ M. I. Dykman $\odot,{ }^{2, \dagger}$ and E. M. Weig $\odot^{1, \hbar}$ \\ ${ }^{1}$ Department of Physics, University of Konstanz, 78457 Konstanz, Germany \\ ${ }^{2}$ Michigan State University, East Lansing, Michigan 48824, USA
}

(Received 19 May 2019; revised manuscript received 21 April 2020; accepted 28 April 2020; published 23 June 2020)

\begin{abstract}
Because of the broken time-translation symmetry, in periodically driven vibrational systems fluctuations of different vibration components have different intensities. Fluctuations of one of the components are often squeezed, whereas fluctuations of the other component, which is shifted in phase by $\pi / 2$, are increased. Squeezing is a multifaceted phenomenon; it attracts much attention from the perspective of highprecision measurements. Here we demonstrate a new and hitherto unappreciated side of squeezing: its direct manifestation in the spectra of driven vibrational systems. With a weakly damped nanomechanical resonator, we study the spectrum of thermal fluctuations of a resonantly driven nonlinear mode. In the attained sideband-resolved regime, we show that the asymmetry of the spectrum directly characterizes the squeezing. This opens a way to deduce squeezing of thermal fluctuations in strongly underdamped resonators, for which a direct determination by a standard homodyne measurement is impeded by frequency fluctuations. The experimental and theoretical results are in excellent agreement. We further extend the theory to also describe the spectral manifestation of squeezing of quantum fluctuations.
\end{abstract}

DOI: 10.1103/PhysRevX.10.021066

\section{INTRODUCTION}

When appropriately scaled, the coordinate and momentum of a vibrational system or their canonical conjugate linear combinations form two vibration components. The scaling is done in such a way that, classically, the components oscillate with equal amplitudes in an isolated system, whereas their phases differ by $\pi / 2$. If the system is coupled to a thermal reservoir, the vibration components fluctuate with the same intensities, in the absence of driving. This is a consequence of the time-translation symmetry, as incrementing the time by a quarter of the oscillation period leads to the interchange of the components (modulo the sign). A periodic driving lifts the symmetry and can result in a reduction of fluctuations of one of the components, the effect of squeezing. Historically, squeezing was first detected in quantum optics [1]. It attracted significant attention, since it can reduce the fluctuations below the quantum limit imposed by the uncertainty principle in the absence of driving [2]. This enables high-precision measurements [3-8]. More recently,

*Present address: Department of Physics, University of Basel, 4056 Basel, Switzerland.

†dykmanm@msu.edu

‡eva.weig@uni-konstanz.de

Published by the American Physical Society under the terms of the Creative Commons Attribution 4.0 International license. Further distribution of this work must maintain attribution to the author(s) and the published article's title, journal citation, and DOI.
Subject Areas: Condensed Matter Physics,

Nanophysics, Nonlinear Dynamics squeezing in the quantum regime was also demonstrated in mechanical systems [9-11].

However, the concept of squeezing of fluctuations in vibrational systems equally applies to the classical regime. Classical squeezing promises to reduce heating in computers [12]; it also represents an important asset for high-precision sensing [13-15] and thus paves the way for a new generation of nanomechanical detectors at room temperature.

Squeezing has been frequently accomplished using parametric pumping or radiation pressure and has been demonstrated and theoretically analyzed for microwave $[16,17]$ and mechanical $[15,18-23]$ resonators as well as for ions in a Penning trap [14]. The classical two-mode squeezing of mechanical resonators by nondegenerate parametric amplification has also been reported [24-26].

Along with parametric oscillators, the other vibrational system intensely studied in different areas, from optics to circuit quantum electrodynamics and to nano- and micromechanical systems, is the Duffing (Kerr) oscillator [27]. This is an oscillator with quartic nonlinearity in the potential. When driven by a resonant field, it can display bistability of forced vibrations. From the time-symmetry argument, one would expect the possibility of fluctuation squeezing in the corresponding vibrational states. A theory of the squeezing was developed in Ref. [28]. However, to date, squeezing in this system has been observed only in a narrow parameter range where the oscillator (a nanomechanical mode) was close to the cusp 
bifurcation point at which the branches of the stable vibrational states merge [29].

Even though squeezing is a feature of one of the vibration components, a natural question is whether the decrease of fluctuations of a particular component is the only manifestation of squeezing. In our experiment we demonstrate that this is not the case. We reveal a different manifestation of squeezing and use it to characterize the squeezing quantitatively. The results demonstrate, in particular, that a driven Duffing oscillator displays strong squeezing in a broad parameter range.

Our approach is based on measuring the spectrum of a resonantly driven vibrational system. The spectra of fluctuations of such a system and of its response to an additional weak field display sideband peaks [30-32]. Such peaks are separated from the peak at the strongdrive frequency and have been seen in micromechanical systems [33]. If the vibrational system is strongly underdamped, the peaks are well resolved. They come from the fluctuations of the amplitude and phase of forced vibrations about their stable values determined by the drive and should have different heights and areas. The asymmetry of the spectrum has been predicted to directly reflect the squeezing [34-36].

In what follows we describe the observation of the sideband-resolved peaks in the fluctuation spectrum of a weakly damped driven nanomechanical resonator. Under sufficiently strong driving the spectrum shows two perfectly resolved peaks symmetrically located on the opposite sides of the driving frequency, but indeed having different heights and areas. The fluctuations are thermal; the resonant periodic drive is the only cause of the system being away from thermal equilibrium. In contrast to the previous experiments, no extra noise or extra drive is added. We use the asymmetry of the spectrum to infer the squeezing and determine the squeezing parameter. Our experimental results agree, with no adjustable parameters, with a theoretical model which extends the one discussed in Refs. [30-32,35].

It is instructive to compare our method with the conventional measurement of a squeezed state. The latter involves the measurement of the individual components (quadratures) of the vibrations, which is accomplished by controlling the phase between the vibrations and an injected signal. The commonly employed method to detect squeezing is a homodyne measurement. This technique has been used in all previous demonstrations of quantum or classical noise squeezing we are aware of, be it the case of a parametric amplifier or a Duffing resonator [1,9-11,14-26,29]. In contrast, our method does not require measuring the individual vibrational components and does not involve homodyne detection. Rather it relies on the simple standard technique of spectral measurements. This is particularly favorable for strongly underdamped resonators, such as the one explored in the present work, as the power spectrum is insensitive to frequency fluctuations as long as they are smaller than the decay rate, while the noise quadratures of weakly damped resonators are difficult to measure independently because of the accumulative effect of frequency fluctuations [37]. To the best of our knowledge, no homodyne measurement of single-mode squeezing of a strongly underdamped mechanical resonator has been reported. The advantage of measuring the power spectrum is not limited to mechanical resonators.

We also use our driven resonator to explore another effect that occurs in nonequilibrium systems with coexisting stable states. For an equilibrium dynamical system such states can be thought of as the minima of a potential in which the system moves. Fluctuations cause switching between the states, forming a distribution over them. For a small fluctuation intensity, the state populations are exponentially different: in an equilibrium system, this difference is given by the Boltzmann factor that contains the difference between the potential minima divided by $k_{B} T$. Only in a narrow range where the minima are of almost equal depth are they almost equally populated, an analog of the firstorder phase transition.

Generically, a nonequilibrium system does not have detailed balance and cannot be mapped onto a Brownian particle in a potential well. Still, it can display an analog of a kinetic phase transition where the state populations are almost equal $[30,38]$. A resonantly driven bistable classical oscillator is a system for which it was predicted where such a transition occurs [30]. Our nanoresonator allows us to find the kinetic phase transition in a system lacking detailed balance and thus to quantitatively test a major aspect of the theory of fluctuations in such systems.

\section{EXPERIMENTAL SYSTEM}

The classical nanomechanical Duffing resonator is realized by a freely suspended silicon nitride string fabricated on a fused silica substrate [39] whose material parameters are reported in literature $[40,41]$. The string under investigation is $270 \mathrm{~nm}$ wide, $100 \mathrm{~nm}$ thick, and $55 \mu \mathrm{m}$ long, like the one depicted in Fig. 1(a). Owing to their strong intrinsic tensile prestress, these nanostring resonators exhibit ultrahigh quality factors of several 100000 at room temperature $[39,42]$. Dielectric transduction combined with a microwave cavity-enhanced heterodyne detection scheme is implemented via two adjacent gold electrodes also apparent in Fig. 1(a). The microwave cavity is pumped on resonance at $\approx 3.6 \mathrm{GHz}$ to enable displacement detection while avoiding unwanted dynamical backaction effects. The application of a dc voltage as well as a near-resonant rf drive tone $V_{\text {in }}$ enables actuation and eigenfrequency tuning of the string $[39,43,44]$. Moreover, the applied dc voltage also affects the damping rate and the nonlinearity, and introduces strong coupling between the out-of-plane and the in-plane mode when tuned into resonance. For all measurements discussed in the following, a constant dc voltage of $5 \mathrm{~V}$ is applied. Under 
(a)

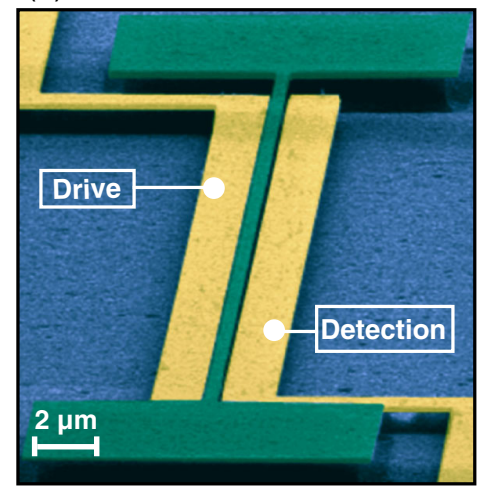

(b)

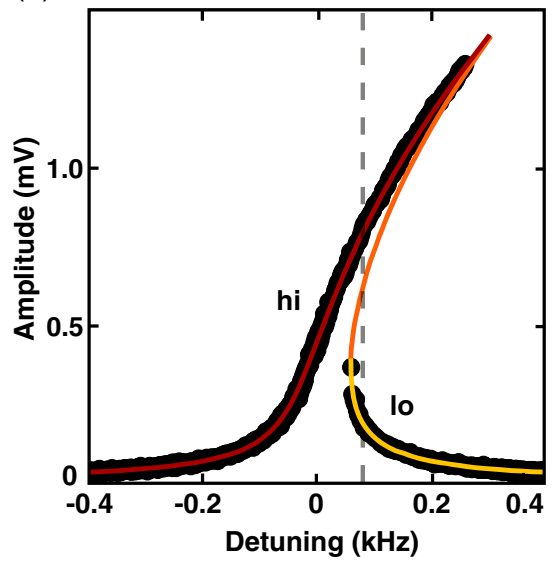

(c)

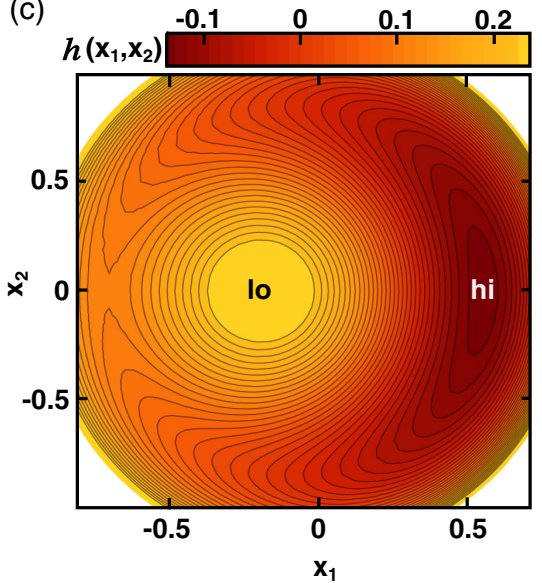

FIG. 1. (a) Scanning electron micrograph of the doubly clamped silicon nitride string resonator (green) and two adjacent gold electrodes (yellow) for dielectric control. Schematic of electronic setup is detailed in the SM [45]. (b) Duffing response curve for an external drive of $-31 \mathrm{dBm}$ (black dots) and fit of the Duffing model [Eq. (1)]. The red (yellow) line denotes the high (low) amplitude solution marked "hi" ("lo"), while the orange line represents the unstable solution. Dashed gray line indicates the theoretically calculated critical switching point. (c) Phase space representation of the effective Hamiltonian function $h\left(x_{1}, x_{2}\right)$. Indicated are the high- ("hi," red) and low- ("lo," yellow) amplitude solution. The Hamiltonian function $h\left(x_{1}, x_{2}\right)$ scaled by $8 \omega_{F} \delta \omega^{2} /(3 \gamma)$ is plotted using the parameter $\sqrt{3} \gamma F^{2} /\left(32 \omega_{F}^{3} \delta \omega^{3}\right)=0.013$, corresponding to the dashed line in $(\mathrm{b})$, whereas the coordinate axes are scaled with $\sqrt{8 \omega_{F} \delta \omega /(3 \gamma)}$.

that condition, the fundamental flexural out-of-plane mode can be considered independently, such that the following analysis is done in the single mechanical mode regime. The experiment is performed under vacuum at a pressure of $\leq 10^{-4}$ mbar and at room temperature of $293 \mathrm{~K}$.

\section{LINEAR REGIME AND CHARACTERIZATION}

The sample is characterized by measuring response curves at various drive powers to calibrate the measurement; see also Sec. II of the Supplemental Material (SM) [45]. A weak drive power allows for the characterization of the system in the linear regime. The frequency response of the resonator is measured as a function of the frequency $f_{F}$ of the applied rf drive. The measured rf voltage signal is proportional to the resonator's amplitude. The resonance of the fundamental out-of-plane mechanical mode is found at $f_{0}=6.529 \mathrm{MHz}$ with a linewidth of $2 \Gamma / 2 \pi=20 \mathrm{~Hz}$, yielding a quality factor of $Q \approx 325000$. Note that this high quality factor is crucial for the presented work as it enables driving the resonator to amplitudes large enough to enter the nonlinear regime and to resolve the satellite peaks appearing in the power spectrum, as discussed in the following.

\section{NONLINEAR REGIME AND DUFFING MODEL}

Increasing the drive power leads to the well-known Duffing response [27,46-49]. In this model the vibration of the single mode is described by the displacement $q(t)$ which obeys the equation

$$
\ddot{q}+2 \Gamma \dot{q}+\omega_{0}^{2} q+\gamma q^{3}=F \cos \left(\omega_{F} t\right)+\xi(t) .
$$

Here, $\omega_{0}=2 \pi f_{0}$ is the angular eigenfrequency, $\Gamma$ the damping rate, $\gamma$ the nonlinearity parameter, $F$ and $\omega_{F}=$ $2 \pi f_{F}$ are the amplitude and frequency of the external driving, and $\xi(t)$ is the thermal noise. The effective mass of the resonator is, for the time being, set to $m=1$. In a stationary vibrational state the coordinate $q(t)=$ $A \cos \left(\omega_{F} t+\theta\right)$ oscillates at the drive frequency with a phase $\theta$ with respect to the drive. The vibration amplitude $A$ is given by the solution of the cubic equation, $A_{j}^{2}\left\{\left[\delta \omega-3 \gamma A_{j}^{2} /\left(8 \omega_{0}\right)\right]^{2}+\Gamma^{2}\right\}=F^{2} / 4 \omega_{0}^{2}$, where $\delta \omega=$ $\omega_{F}-\omega_{0}$ is the frequency detuning, $|\delta \omega| \ll \omega_{0}$ for the considered near-resonant driving. The Duffing equation reflects the fact that the vibration frequency of a nonlinear resonator depends on its amplitude. It can have one or three positive solutions. In the latter case, only the solutions with the largest and the smallest amplitude, $A_{\text {hi }}$ and $A_{\mathrm{lo}}$, are stable. An example of the measured amplitude as a function of the frequency detuning $\delta \omega$ is shown in Fig. 1(b) by black dots. The solid line represents a fit of the Duffing model [Eq. (1)].

Only one fitting parameter, the Duffing nonlinearity parameter $\gamma$, is required, since the eigenfrequency $\omega_{0}$, the damping rate $\Gamma$, as well as the calibration of the driving force $F$ are known from the characterization in the linear regime [45]. The nonlinear response curves obtained for different values of the $\mathrm{rf}$ drive power $P=-31 \mathrm{dBm}$ [shown in Fig. 1(b)], $-30 \mathrm{dBm}$ (shown in the SM [45]), $-25 \mathrm{dBm},-20 \mathrm{dBm}$, and $-18 \mathrm{dBm}$ are all fit using a single value of $\gamma$. As the amplitude of the resonator is 
measured in volts, the fit yields a nonlinearity parameter in units of $\mathrm{V}^{-2} \mathrm{~s}^{-2}$. The obtained value, $9.28 \times 10^{16} \mathrm{~V}^{-2} \mathrm{~s}^{-2}$, can be converted into $\gamma=1.54 \times 10^{26} \mathrm{~m}^{-2} \mathrm{~s}^{-2}$ using the amplitude conversion procedure described in the SM [45].

\section{THEORY: SQUEEZING IN THE POWER SPECTRUM OF A WEAKLY DAMPED OSCILLATOR}

The theoretical analysis of the resonator dynamics is done by switching to the rotating frame, $q(t)=x_{1}(t) \cos \left(\omega_{F} t\right)+$ $x_{2}(t) \sin \left(\omega_{F} t\right) \quad$ and $\quad \dot{q}(t)=-\omega_{F}\left[x_{1}(t) \sin \left(\omega_{F} t\right)-\right.$ $\left.x_{2}(t) \cos \left(\omega_{F} t\right)\right]$, where the quadratures $x_{1}(t)$ and $x_{2}(t)$ are new conjugate variables. Using the standard rotating wave approximation, one finds that the time evolution of these variables is described by the equations

$$
\begin{gathered}
\dot{x}_{1}=\frac{\partial h\left(x_{1}, x_{2}\right)}{\partial x_{2}}-\Gamma x_{1}, \\
\dot{x}_{2}=-\frac{\partial h\left(x_{1}, x_{2}\right)}{\partial x_{1}}-\Gamma x_{2},
\end{gathered}
$$

with the Hamiltonian function

$h\left(x_{1}, x_{2}\right)=\frac{3 \gamma}{32 \omega_{F}}\left(x_{1}^{2}+x_{2}^{2}\right)^{2}-\frac{\delta \omega}{2}\left(x_{1}^{2}+x_{2}^{2}\right)-\frac{F}{2 \omega_{F}} x_{1}$.

In writing Eqs. (2) and (3) we have, for the time being, disregarded the noise. A contour plot of the function $h\left(x_{1}, x_{2}\right)$ in the range of the bistability is shown in Fig. 1(c).

A remarkable feature of our high- $Q$ nanostring resonator is that the damping rate $\Gamma$ is small not only compared to the eigenfrequency $\omega_{0}$, but also compared to the frequency detuning $\delta \omega$ and/or the typical frequency change due to the nonlinearity $\gamma A_{j}^{2} / \omega_{F}$. Therefore the damping can be treated as a small perturbation of the Hamiltonian dynamics of an auxiliary "particle" with coordinate $x_{1}$ and momentum $x_{2}$. In this limit of weak damping, the extrema $j=$ hi, lo of $h$ correspond to the two stable states of forced vibrations [50]. At the extrema, $x_{2, j}=0$, whereas $\left|x_{1, j}\right|=A_{j}$ gives the vibration amplitude, if one disregards corrections $\propto \Gamma^{2}$. The Hamiltonian dynamics for $\Gamma=0$ is characterized by the frequency $\omega_{j}$ of small-amplitude vibrations about the extrema of $h\left(x_{1}, x_{2}\right)$,

$$
\omega_{j}=\sqrt{\omega_{j}^{(1)} \omega_{j}^{(2)}}
$$

where $\omega_{j}^{(1)}=3 \gamma A_{j}^{2} / 8 \omega_{F}-\delta \omega$ and $\omega_{j}^{(2)}=9 \gamma A_{j}^{2} / 8 \omega_{F}-\delta \omega$ (we note that $\omega_{j}^{(1,2)}$ can be positive or negative, but their product is positive). The frequency is different in the highand low-amplitude states. In the considered weak-damping case, $\Gamma \ll \omega_{j}$.
We now reintroduce noise into the equations for the quadratures and discuss thermal fluctuations about the stable states. Even though the nanoresonator under investigation is small, thermal fluctuations at room temperature are weak. If there is no driving [ $F=0$ in Eq. (4)], clearly $\left\langle x_{1}\right\rangle=\left\langle x_{2}\right\rangle=0$, while the mean-square values of the quadratures are the same, and for the considered weak nonlinearity $\left\langle x_{1}^{2}\right\rangle=\left\langle x_{2}^{2}\right\rangle=k_{B} T / \omega_{0}^{2}$.

To analyze the squeezing of fluctuations about the states of forced vibrations for the case of weak damping, we linearize the equations of motion about the stable vibrational states $\left(x_{1 j}, x_{2 j}\right)$ keeping the lowest-order terms in the decay rate $\Gamma$ (such linearization may be insufficient in the case of extremely weak damping, as discussed in Sec. I.E of the SM [45]). From Eqs. (1)-(3), the resulting equations for the increments $\delta x_{1,2}$ in the presence of noise are

$$
\begin{gathered}
\delta \dot{x}_{1}=\omega_{j}^{(1)} \delta x_{2}-\Gamma\left(1+\mu_{j}\right) \delta x_{1}+\xi_{x_{1}}(t), \\
\delta \dot{x}_{2}=-\omega_{j}^{(2)} \delta x_{1}-\Gamma\left(1-\mu_{j}\right) \delta x_{2}+\xi_{x_{2}}(t) .
\end{gathered}
$$

Here, $\mu_{j}=6 \gamma A_{j}^{2} /\left(3 \gamma A_{j}^{2}-8 \omega_{F} \delta \omega\right)$ and we have disregarded terms $\propto \Gamma^{2}$. Functions $\xi_{x_{1}}(t)$ and $\xi_{x_{2}}(t)$ describe the noise that drives the quadratures. In the phenomenological model Eq. (1) these functions are given by the real and imaginary parts of $i \xi(t) \exp \left(i \omega_{F} t\right) / \omega_{F}$. If the noise comes from the same coupling to a thermal bath that leads to the vibration decay, on the timescale $\gg \omega_{F}^{-1}$ it is zero mean, Gaussian and $\delta$ correlated, and the components $\xi_{x_{1}}, \quad \xi_{x_{2}}$ are independent and have equal intensity, $\left\langle\xi_{x_{1}}(t) \xi_{x_{1}}(0)\right\rangle=\left\langle\xi_{x_{2}}(t) \xi_{x_{2}}(0)\right\rangle=\left(2 \Gamma k_{B} T / \omega_{F}^{2}\right) \delta\left(t-t^{\prime}\right)$. The power spectrum of the fluctuations of the oscillator coordinate in the approximations (6) and (7) is given by Eq. (S13) of the SM [45].

A qualitative feature of the driven resonator is that the mean-square fluctuations of the in-phase and quadrature components of the coordinate are no longer equal and, for one of them, can be smaller than in the absence of the drive. This is the squeezing effect. In the considered case where the vibrations in the rotating frame are weakly damped, the mean-square fluctuations in the state $j$ are (see the SM [45])

$$
\begin{aligned}
& \left\langle\delta x_{1}^{2}\right\rangle_{j}=\frac{k_{B} T}{2 m \omega_{F}^{2}}\left(1+e^{-4 \varphi_{j}}\right), \\
& \left\langle\delta x_{2}^{2}\right\rangle_{j}=\frac{k_{B} T}{2 m \omega_{F}^{2}}\left(1+e^{4 \varphi_{j}}\right),
\end{aligned}
$$

where the expression

$$
\exp \left(4 \varphi_{j}\right)=\omega_{j}^{(2)} / \omega_{j}^{(1)}
$$

defines the squeezing parameter $\varphi_{j}$. Here, we have reintroduced the effective mass of the nanoresonator $m$ to facilitate 
the comparison with the experiment. In the absence of driving, we find $A_{j}=0$ and thus $\varphi_{j}=0$, such that we recover the equipartition theorem, $\left\langle\delta x_{1}^{2}\right\rangle=\left\langle\delta x_{2}^{2}\right\rangle$. For the large-amplitude stable state $\varphi_{j} \equiv \varphi_{\mathrm{hi}}>0$, whereas for the small-amplitude state $\varphi_{j} \equiv \varphi_{\mathrm{lo}}<0$. Obviously, the maximum squeezing attainable is a $50 \%$ reduction of the squeezed quadrature according to Eqs. (8) and (9).

Remarkably, the squeezing appears directly in the power spectrum of the resonator [34,35]. In the weak-damping limit $\Gamma \ll \omega_{j}$, one obtains

$$
\begin{aligned}
Q_{j}(\omega) \approx & \frac{\Gamma k_{B} T}{4 \pi m \omega_{F}^{2}} \frac{\cosh 2 \varphi_{j}\left(\cosh 2 \varphi_{j} \pm 1\right)}{\left(\omega-\omega_{F} \mp \mathcal{S}_{j} \omega_{j}\right)^{2}+\Gamma^{2}} \\
& \text { for }\left|\omega-\omega_{F} \mp \mathcal{S}_{j} \omega_{j}\right| \ll \omega_{j},
\end{aligned}
$$

with $\mathcal{S}_{\text {hi }}=+1$ for the large-amplitude stable state and $\mathcal{S}_{\text {lo }}=-1$ for the small-amplitude stable state, respectively [45]. The power spectrum $Q_{j}(\omega)$ consists of two Lorentzian peaks centered at the frequencies $\omega_{F} \pm \mathcal{S}_{j} \omega_{j}$ with the half-width given by the damping rate of resonator in the absence of driving $\Gamma$. They can be thought of as the Stokes and anti-Stokes components of the Raman scattering of the driving field by the small-amplitude vibrations of the resonator near the corresponding stable state. Importantly, the very state is formed by the drive. The ratio of the intensities of the satellite peaks,

$$
\begin{gathered}
\mathcal{I}_{\mathrm{hi}}^{(+)} / \mathcal{I}_{\mathrm{hi}}^{(-)}=1 / \tanh ^{2}\left(\varphi_{\mathrm{hi}}\right), \\
\mathcal{I}_{\mathrm{lo}}^{(+)} / \mathcal{I}_{\mathrm{lo}}^{(-)}=\tanh ^{2}\left(\varphi_{\mathrm{lo}}\right),
\end{gathered}
$$

is determined by the squeezing parameter $\varphi_{j}$. The squeezing parameter can thus be directly found from the power spectrum. An advantageous feature of the ratios Eqs. (12) and (13) is their independence of the temperature. Therefore, even if the nanoresonator is slightly heated by the drive, they should not change.

We emphasize that the peak intensities $\mathcal{I}^{( \pm)}$are well defined if the satellite peaks are well resolved. This condition is met, as seen from Eq. (11), provided the widths of the peaks are small compared with the distance between them, i.e., $\Gamma \ll \omega_{j}$. The latter inequality has a simple physical meaning: in the rotating frame, the vibrations about the stable state of forced oscillations of the nonlinear resonator are underdamped; see also Secs. I.D and I.E of the SM [45]. This is a stronger condition than the condition that the nanoresonator mode is underdamped in the laboratory frame, i.e., $\Gamma \ll \omega_{0}$. However, for weakly damped nonlinear nanoresonators of current interest, including the one studied in this paper, the condition $\Gamma \ll \omega_{j}$ holds in a broad range of the amplitudes and frequencies of the driving field.

The relations (12) and (13) do not hold in the quantum regime, $\hbar \omega_{0} \gtrsim k_{B} T$. The power spectrum (i.e., the fluorescence spectrum) is symmetric with respect to the drive frequency for $k_{B} T \ll \hbar \omega_{0}$ [31]. However, quantum fluctuations of the driven nonlinear mode are squeezed. The variances of the in-phase and quadrature components are different. In the strongly underdamped regime discussed here, there is an alternative spectral measurement that allows one to find the squeezing parameter both in the classical and quantum regimes. This measurement involves driving the mode by an additional weak probe drive $F^{\prime} \exp \left(-i \omega^{\prime} t\right)$ at frequency $\omega^{\prime}$ close to the strong-drive frequency $\omega_{F}$. Such drive leads to an additional term in the mode displacement, which oscillates at frequencies $\omega^{\prime} \quad$ and $\quad 2 \omega_{F}-\omega^{\prime}, \quad \delta\langle q(t)\rangle=\chi\left(\omega^{\prime}\right) F^{\prime} \exp \left(-i \omega^{\prime} t\right)+$ $\mathcal{X}\left(\omega^{\prime}\right) F^{\prime} \exp \left[-i\left(2 \omega_{F}-\omega^{\prime}\right) t\right][30,32]$. In a nanomechanical resonator, spectral peaks at the frequency of the probe drive have been observed in Ref. [51]. The susceptibility $\chi(\omega)$ directly reveals the squeezing in the strongly underdamped regime. Both $\operatorname{Im} \chi(\omega)$ and $|\chi(\omega)|^{2}$ display two narrow sideband peaks, with the ratio of their areas determined by the squeezing parameter [45]. Squeezing of quantum fluctuations about a metastable state occurs also in a driven oscillator resonantly coupled to a two-level system [52].

\section{EXPERIMENTAL OBSERVATION OF THE THERMAL SQUEEZING IN THE POWER SPECTRUM}

To validate these theoretical findings, we apply a resonant sinusoidal drive tone to the fundamental flexural mode of the nanostring $\left(f_{F}=f_{0}\right)$ and record power spectra for different drive powers using a spectrum analyzer operated in the FFT mode. Under resonant driving, the resonator has one stable vibrational state, with the parameters in Eqs. (5)-(11) corresponding to the high-amplitude state $A_{\text {hi }}$. Figure 2(a) displays power spectra for drive powers in the range between -45 and $-5 \mathrm{dBm}$, with a color-coded signal power $(\mathrm{dBm})$. The bright, narrow line centered at zero corresponds to forced vibrations at $f_{F}$. The drive tone is flanked by two satellite peaks. Their separation from the drive tone is symmetric and increases with drive power. We identify these sideband-resolved satellite peaks with the thermal noise-induced small-amplitude vibrations around the stable state of the driven resonator. Thus the peaks should be centered at the frequencies $\omega_{F} \pm \omega_{\mathrm{hi}}$.

The experimentally observed satellite peaks are compared with the theoretical prediction of Eq. (5) in Fig. 2(a), where the calculated positions of the peaks are shown as open red circles. For better visualization, only a few distinct points are plotted. We find the experimental data to coincide with the theory, and also recover the expected scaling of the splitting of the satellite peaks with the drive power $\omega_{\mathrm{hi}} \propto A_{\mathrm{hi}}^{2} \propto F^{2 / 3} \propto P^{1 / 3}$.

Another remarkable feature of the satellite peaks is apparent from their intensities. Figure 2(b) depicts a line cut extracted from Fig. 2(a) at $-20 \mathrm{dBm}$. Each peak is fitted 
(a)

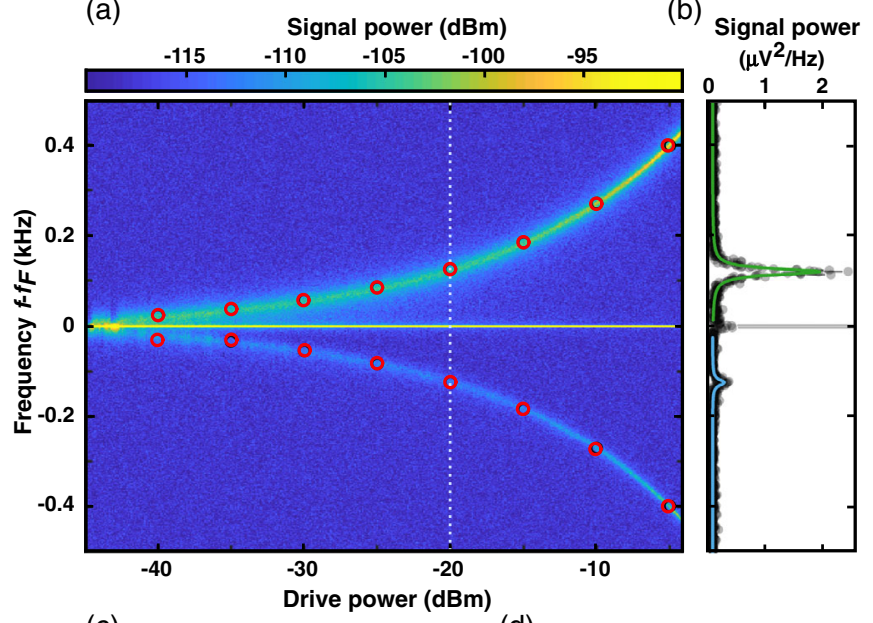

(c)
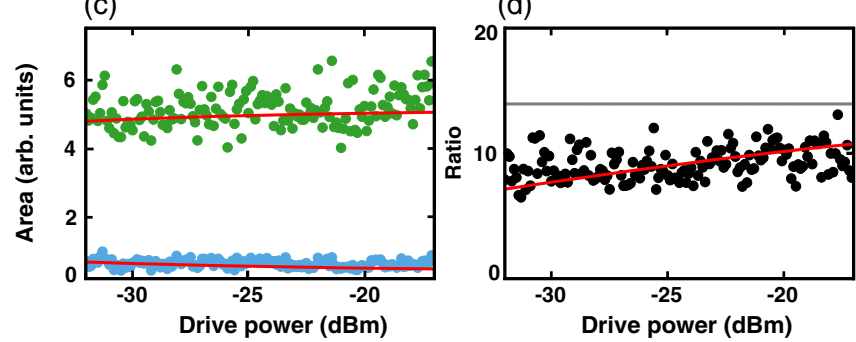

FIG. 2. (a) Color-coded power spectra showing the increasing splitting of the satellite peaks with the increasing drive power for the drive frequency $f_{F}=f_{0}$, where the resonator is monostable. Red open circles denote the calculated positions of the satellite peaks. The central line at $f-f_{F}=0$ is plotted with a reduced brightness to improve the visibility of the satellites. (b) Line cut along the white dotted line in (a) illustrating the satellite peaks as well as their Lorentzian fits for a drive of $-20 \mathrm{dBm}$. The central line at $f=f_{F}$ (gray line) is truncated. (c) Area of the high(green) and low- (blue) frequency satellite peaks extracted from the Lorentzian fits as a function of the drive power. Red lines show the theoretical prediction that takes into account the partial overlap of the peaks. (d) Ratio of the areas of the satellite peaks as a function of drive power. Red and gray lines show, respectively, the theoretical prediction that takes into account the partial overlap of the peaks (see SM [45]) and the one based on Eq. (12).

by a Lorentzian with a linewidth of $2 \Gamma / 2 \pi=20 \mathrm{~Hz}$, as shown in Fig. 2(b). As predicted by the theoretical model, this linewidth coincides with that of the linear resonance of the string [45]. Clearly, the satellite peak at higher frequency is much brighter than that at the lower frequency. This observation is in agreement with the theoretical model, which predicts nonequal intensities of the satellite peaks as a result of the classical squeezing of thermal fluctuations.

More precisely, as outlined in Eq. (12) for the highamplitude state $A_{\mathrm{hi}}$, a higher intensity is expected for the satellite peak at the higher frequency. Following the model, the ratio of the areas enclosed by the peaks is simply related to the squeezing parameter $\varphi_{j}$. The areas extracted from the fit are plotted in Fig. 2(c) as a function of the drive power, where green corresponds to the brighter, higher-frequency
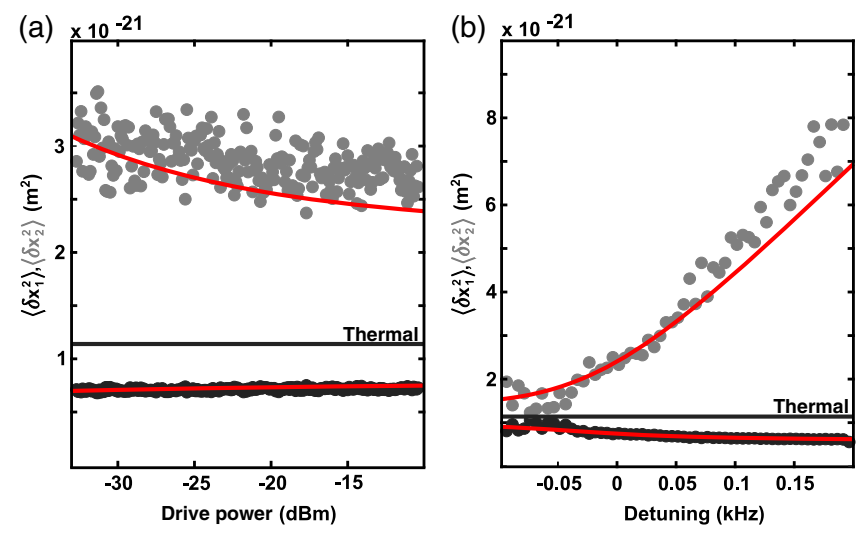

FIG. 3. Variance of in-phase and quadrature fluctuations around the stable state of forced vibrations as a function of (a) drive power and (b) detuning. Black and gray dots show the in-phase and quadrature values extracted from the experimentally determined satellite area ratio, respectively, whereas red lines show the corresponding theoretical model including the partial overlap of the peaks (no free parameters; see SM [45]). Black lines in (a) and (b) indicate the thermomechanical fluctuations at $293 \mathrm{~K}$.

peak and blue to the lower-frequency peak. The experimental data are compared with the theoretical predictions which are shown in Fig. 2(c) by the red lines [45]. As suggested by the theoretical model, a pronounced difference in the areas is observed. The ratio of the areas is plotted in Fig. 2(d), and again, we find very good agreement between the experimental data (black dots) and the theoretical predictions (red line).

The theoretical calculations of the areas and their ratio shown in Fig. 2 are obtained from a more general analysis of the power spectrum. This analysis is not limited to the condition $\Gamma \ll \omega_{j}$ and thus takes into account the overlapping of the satellite peaks. It is provided in Secs. I C and I D of the SM [45]. The ratio of the areas for the limit of small damping, Eqs. (12) and (13), is also included in Fig. 2(d) as a gray line. In this limit the ratio is independent of the drive power; it provides the fundamental limiting value for the ratio of the areas of the satellite peaks. For our high- $Q$ nanostring resonator, the measured ratio approaches this value as the separation of the peaks increases with the increasing drive power.

The squeezing parameter $\varphi_{\mathrm{hi}}$ extracted from the areas of the satellites discussed in Figs. 2(c) and 2(d) can be employed to compute the mean-square fluctuations of the in-phase and quadrature component of the stable state of forced vibrations using Eqs. (8) and (9). Figure 3(a) compares the experimentally obtained fluctuations $\left[\left\langle\delta x_{1}^{2}\right\rangle_{\mathrm{hi}}\right.$ and $\left\langle\delta x_{2}^{2}\right\rangle_{\text {hi }}$ represented as black (gray) dots, respectively] with the theoretical model accounting for the partial overlap of the satellite peaks [45] (red lines). The mean square of the thermomechanical fluctuations at $293 \mathrm{~K}$ is included as a black solid line, clearly showing that a significant squeezing of the in-phase quadrature is accomplished. 
According to the theory, the satellite peaks in the power spectrum also depend on the detuning of the drive frequency $f_{F}-f_{0}$. We therefore repeat the measurement routine, now for a fixed drive power of $-20 \mathrm{dBm}$ and a variable detuning of the drive. The resonator is initialized in the high-amplitude state by sweeping up the drive frequency from $30 \mathrm{kHz}$ below $f_{0}$ to the desired $f_{F}$ before recording the power spectrum.

Figure 4(a) displays the power spectra as a function of the detuning $f_{F}-f_{0}$. For large negative detuning, $f_{F}-f_{0}<0$, only the satellite peak at a higher frequency can be discerned; its distance from the drive tone $f_{F}$ increases with the increasing $-\left(f_{F}-f_{0}\right)$. For small detuning, both satellite peaks are resolved. They are at equal distances from $f_{F}$, which only slightly increase with $f_{F}-f_{0}$ for $f_{F}-f_{0}>0$. In contrast, the intensities of the peaks are increasing. The splitting at zero detuning equals the one shown in the resonantly driven case discussed in Fig. 2(a) for a drive power of $-20 \mathrm{dBm}$.

Interestingly, the satellite peak at higher frequency vanishes abruptly for the detuning of $190 \mathrm{~Hz}$, whereas the lower frequency one remains. However, the lowerfrequency peak exhibits a discontinuity at $190 \mathrm{~Hz}$, and continues with a larger splitting, a different slope, and a strongly reduced intensity. At the same detuning of $190 \mathrm{~Hz}$ the amplitude at the drive tone drops to a drastically smaller value, as shown in Fig. 4(b). This is a signature of the resonator switching from the high-amplitude state $A_{\text {hi }}$ to the low-amplitude state $A_{\mathrm{lo}}$. The displayed signal power has been extracted from a line cut in Fig. 4(a) at the driving frequency, $f=f_{F}$. Since the measurement routine to record each of the power spectra in Fig. 4(a) exposes the resonator to the drive for more than one minute, this represents a much slower measurement than a typical
(Duffing) response curve measurement, such as the one shown in Fig. 1(b).

The observed satellite peaks on the opposite sides of the critical detuning $\Delta f_{\text {cr }} \equiv\left(f_{F}-f_{0}\right)_{\text {cr }} \approx 190 \mathrm{~Hz}$ are associated with the high- and low-amplitude state $A_{\mathrm{hi}}$ and $A_{\mathrm{lo}}$ of the resonator. They are compared in Fig. 4(a) with the theoretical prediction for the two stable states, which are superposed on the measured data as red and yellow open circles, respectively. In both states, we find the experiment and the theory to coincide completely.

We repeat the analysis described for the resonantly driven case of Fig. 2 and extract the areas of the highand low-frequency satellite peaks for each power spectrum by fitting two Lorentzians (not shown). When the resonator is in the high-amplitude state, i.e., for a detuning below $\Delta f_{\text {cr }}$, both satellite peaks are resolved and appear for a certain range of detunings. The ratio of the obtained areas of the peaks for this detuning is shown in Fig. 4(c) as black dots. Like for the resonantly driven case, this quantity is associated with the squeezing parameter.

According to the theory of Sec. V, the ratio of the areas of the peaks depends on the detuning of the drive frequency. For the high-amplitude stable state, it is asymmetric with respect to $f_{0}$ and decreases as the detuning varies from negative to positive. The experimental data in Fig. 4(c) are compared with the theoretical prediction for the weakdamping limit, Eq. (12) (gray line), and for the more general approximation that takes into account the small overlapping of the satellite peaks [45] (red line). Once more, the agreement between the experiment and the theory is remarkable. The resulting mean-square fluctuations of the in-phase and quadrature component about the stable state of forced vibrations are presented in Fig. 3(b), again

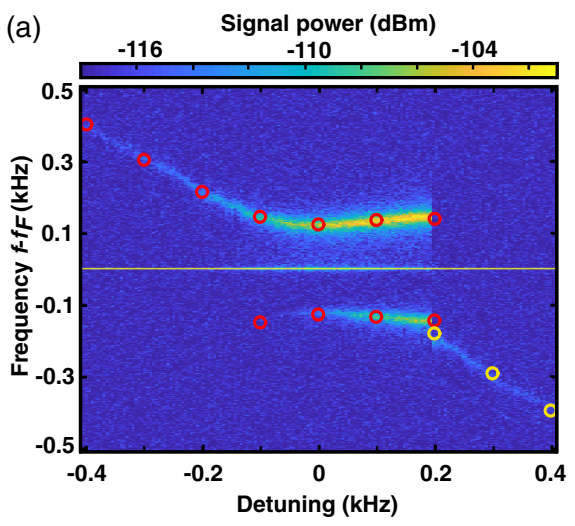

(b)

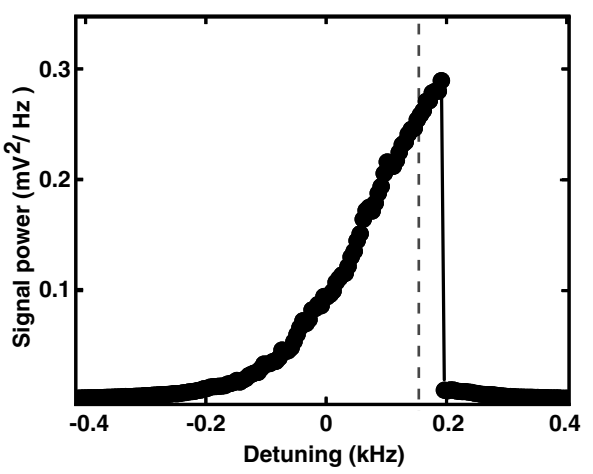

(c)

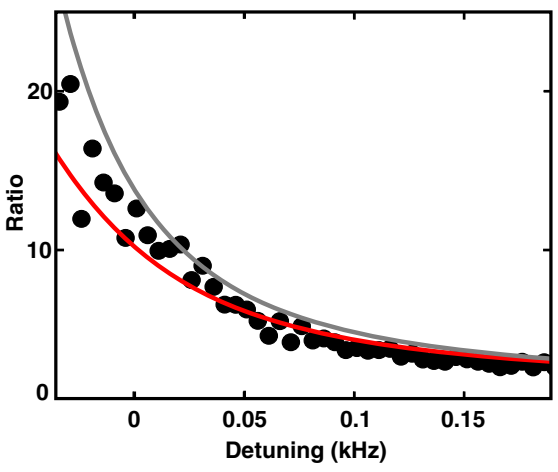

FIG. 4. (a) Color-coded power spectra showing the positions and intensities of the satellite peaks as a function of the detuning of the drive frequency $f_{F}-f_{0}$ for the drive power $-20 \mathrm{dBm}$. The central line at $f-f_{F}=0$ is plotted with a reduced brightness to improve the visibility of the satellites. Open circles denote the calculated positions for the high- (red) and low- (yellow) amplitude states [see Fig. 1(b)]. (b) The power of the signal at the drive frequency $f_{F}$ as a function of the detuning. The discontinuity observed at a detuning of $190 \mathrm{~Hz}$ indicates the switching of the resonator from the high- to the low-amplitude state for a slow ramp-up of the detuning. It coincides with the discontinuity of the satellite peaks in (a). The dashed gray line indicates the theoretically calculated critical switching point. (c) Ratio of the areas of the satellite peaks for the high-amplitude state as a function of the detuning. Red and gray lines show the theoretical predictions with and without the small overlapping of the satellite peaks, respectively. 
demonstrating the squeezing of the in-phase quadrature with respect to the thermomechanical fluctuations.

Above the switching point, $f_{F}-f_{0}>\Delta f_{\text {cr }}$, the resonator is in the low-amplitude state, and only one satellite peak is resolved. Therefore the ratio of the areas of the peaks and thus the squeezing parameter cannot be evaluated here. Notice, however, that the data clearly show the anticipated reversal of the intensities of the satellite peaks between the two stable solutions, as predicted by Eqs. (12) and (13): While the high-frequency satellite peak has a higher intensity for the high-amplitude stable state, the lowfrequency peak is the dominating one for the low-amplitude state. In addition, while the ratio of the areas of the peaks for the high-amplitude state has decreased to a value $\approx 1$ in the vicinity of the switching point $f_{\text {cr }}$, for the lowamplitude state the ratio is large, according to the theory, which explains why the low-frequency satellite peak is resolved whereas the high-frequency peak cannot be detected.

For a positive or negative detuning exceeding $400 \mathrm{~Hz}$, Fig. 4(a) exhibits only one peak, and the slope of its frequency versus the drive frequency is -1 . Such slope and a single peak in the power spectrum are expected for an oscillator in the absence of a driving force. Experimentally, for still larger detuning, we are not able to resolve thermal motion of the driven resonator, as is also the case for the undriven resonator. We attribute this to an insufficient displacement sensitivity of the detection setup far away from the driving frequency or in the absence of the drive, while the thermally induced spectral features are resolved near $f_{F}$. Apparently, the displacement sensitivity increases in the presence of the driving, which is likely a consequence of our heterodyne microwave-cavity assisted displacement detection scheme [39].

\section{CRITICAL SWITCHING POINT}

Finally, we discuss the switching between the two stable states of the Duffing resonator. It is characterized by two rates, that from the high-amplitude to the low-amplitude state, $W_{\text {hi } \rightarrow \text { lo }}$, and that from the low-amplitude to the highamplitude state, $W_{\mathrm{lo} \rightarrow \mathrm{hi}}$. At the critical frequency detuning these rates are equal, $W_{\mathrm{hi} \rightarrow \mathrm{lo}}=W_{\mathrm{lo} \rightarrow \mathrm{hi}}$. Respectively, the stationary populations of the stable states are also equal. The rates change with the parameters exponentially strongly. Therefore, away from the critical value of the detuning, the populations of the states are strongly different and only one state is "visible." If the detuning is slowly varied across the critical value, the oscillator should switch from one state to the other in a very narrow range. For weak damping, $\Gamma \ll \delta \omega$, the theoretical value of the critical detuning [30], in terms of the parameters of the studied nanoresonator, is $\Delta f_{\text {cr }} \approx 904.6 \mathrm{~s}^{-1}\left(V_{\text {in }}[V]\right)^{2 / 3}$. It is shown in Fig. 1(b) as a vertical dashed gray line at $V_{\text {in }}=$ $17.8 \mathrm{mV}(-31 \mathrm{dBm})$.
Experimentally, the interchange of the most probable states at the critical point can only be observed in a slow measurement. Clearly, the response curve shown in Fig. 1(b) does not reveal this point, since the detuning was swept in both directions fast enough to allow the system to stay in the metastable high- or low-amplitude state well beyond the critical point, until close to the bifurcation point.

In contrast, the response curve shown in Fig. 4(b) results from a much slower measurement, as described above. This allows the resonator to approach its most probable stable state for every applied detuning and clearly demonstrates sharp switching between occupying practically one or the other state.

The switching point observed in Fig. 4(b) is expected to be close to the theoretical critical switching point, which is shown by a dashed gray line $\left(V_{\text {in }}=65 \mathrm{mV}\right)$. Indeed, the difference between the experimental and theoretical values is only $40 \mathrm{~Hz}$. This difference can be attributed to a slight nonadiabaticity of the frequency sweep. Futhermore, given the statistical nature of the switching, slow room temperature fluctuations cannot be ruled out as an alternative source of the discrepancy, because the effect of a typical eigenfrequency drift of almost $1 \mathrm{kHz} / \mathrm{K}$ could not be completely eliminated, even though the eigenfrequency was redetermined prior to every measurement. In the future, the results can be extended to measure the individual switching rates using different sweep times [53-55].

We emphasize that, in the regime we have studied, the driven resonator has no detailed balance. Understanding fluctuation-induced transitions between the stable states of systems lacking detailed balance, i.e., generically, for all systems away from thermal equilibrium, is of interest for various areas of physics, chemistry, and biology. The weakdamping regime attained in the present work is particularly important, as the phase space of the system is two dimensional rather than the effectively one-dimensional phase space close to bifurcation points. A high-dimensional phase space significantly complicates the theoretical analysis of the switching rate. To the best of our knowledge, the present results show the first quantitative comparison with analytical results obtained for systems lacking detailed balance.

\section{CONCLUSIONS}

In conclusion, we report a new manifestation of squeezing of thermal fluctuations in a broad parameter range of a resonantly driven nanomechanical mode. The squeezing is indirectly determined by measuring the power spectrum of the mode in the sideband-resolved regime, where the spectrum exhibits two well-separated peaks symmetrically positioned with respect to the drive frequency. The peaks can be thought of as Stokes and anti-Stokes component in a Raman scattering picture with the caveat that the 
underlying process is multiphoton, as multiple photons of the resonant driving field are involved.

The sidebands feature unequal intensities. The ratio of the intensities is determined by the squeezing parameter. It was directly read out from the experimental data, thus providing a novel way not only to infer but also to quantitatively characterize squeezing.

Our findings are supported by a theoretical model which is in excellent agreement with the experimental data with no free parameters. The model shows that, for the resonantly driven underdamped Duffing resonator, the squeezed quadrature can be suppressed by a factor of 2 , giving rise to a $3 \mathrm{~dB}$ limit, as in the case of parametrically induced squeezing [18]. Importantly, no fine-tuning to a specific operation point is required for obtaining squeezing in a high-quality-factor resonator.

Squeezing of thermal fluctuations about the state of forced vibrations in weakly damped nonlinear systems is a generic concept as it is related to the breaking of the continuous time-translation symmetry by the drive. The same applies to the asymmetry of the power spectrum and the response spectrum. Therefore, the squeezing and the asymmetry are intrinsically related to each other and we use one of them to characterize the other.

At the same time, it should be noted that the spectral characterization of the squeezing is an indirect one. For applications in precision sensing, care should be taken to ensure that no extra noise is added by the measurement setup.

An important advantageous feature of characterizing squeezing of thermal (and quantum) fluctuations in driven mesoscopic vibrational systems from a spectral measurement is its insensitivity to weak-frequency noise. This is important both for nanomechanical resonators, as the ones studied here, and also for microwave cavity modes. In these systems, the mode eigenfrequencies display slow fluctuations with $1 / f$ type spectrum. Such fluctuations lead to a small broadening of the spectral peaks and a very small change of the peak intensities. Thus they make a small effect on the measured squeezing parameter. In contrast, they significantly complicate the homodyne measurement for weakly damped systems, as discussed in Sec. II.F of the SM [45].

A promising application is the possibility of employing driven weakly damped modes as detectors of weak signals at frequency $f_{S}$ close to the drive frequency, $\left|f_{F}-f_{S}\right| \approx \omega_{j}$. Driven modes can resonantly amplify such signals, which can be thought of as a multiphoton analog of stimulated Raman scattering. The amplification is determined by the squeezing [45], which in turn allows one to determine the squeezing parameter from the response spectrum. Importantly, the corresponding spectral measurement can be done also in the quantum regime [45], where, as shown in Ref. [31], the sidebands in the emission spectrum are symmetric independent of the squeezing parameter.

Data and analysis code are available at [56].

\section{ACKNOWLEDGMENTS}

Financial support by the Deutsche Forschungsgemeinschaft via the collaborative research center SFB 767, the European Union's Horizon 2020 Research and Innovation Programme under Grant Agreement No. 732894 (FET Proactive HOT), and the German Federal Ministry of Education and Research (Contract No. 13N14777) within the European QuantERA co-fund project QuaSeRT is gratefully acknowledged. M. I. D. also acknowledges support from the Zukunftskolleg Senior Fellowship at the University of Konstanz and from the National Science Foundation (Grant No. DMR-1806473).

[1] R. E. Slusher, L. W. Hollberg, B. Yurke, J. C. Mertz, and J. F. Valley, Observation of Squeezed States Generated by Four-Wave Mixing in an Optical Cavity, Phys. Rev. Lett. 55, 2409 (1985).

[2] D. F. Walls, Squeezed States of Light, Nature (London) 306, 141 (1983).

[3] C. M. Caves, Quantum-Mechanical Noise in an Interferometer, Phys. Rev. D 23, 1693 (1981).

[4] J. Aasi et al., Enhanced Sensitivity of the LIGO Gravitational Wave Detector by Using Squeezed States of Light, Nat. Photonics 7, 613 (2013).

[5] J. Abadie et al. (The LIGO Scientific Collaboration), A Gravitational Wave Observatory Operating beyond the Quantum Shot-Noise Limit, Nat. Phys. 7, 962 (2011).

[6] M. Tse et al., Quantum-Enhanced Advanced LIGO Detectors in the Era of Gravitational-Wave Astronomy, Phys. Rev. Lett. 123, 231107 (2019).

[7] F. Acernese et al., Increasing the Astrophysical Reach of the Advanced Virgo Detector via the Application of Squeezed Vacuum States of Light, Phys. Rev. Lett. 123, 231108 (2019).

[8] M. Malnou, D. A. Palken, B. M. Brubaker, L. R. Vale, G. C. Hilton, and K.W. Lehnert, Squeezed Vacuum Used to Accelerate the Search for a Weak Classical Signal, Phys. Rev. X 9, 021023 (2019).

[9] E. E. Wollman, C. U. Lei, A. J. Weinstein, J. Suh, A. Kronwald, F. Marquardt, A. A. Clerk, and K. C. Schwab, Quantum Squeezing of Motion in a Mechanical Resonator, Science 349, 952 (2015).

[10] F. Lecocq, J. B. Clark, R. W. Simmonds, J. Aumentado, and J. D. Teufel, Quantum Nondemolition Measurement of a Nonclassical State of a Massive Object, Phys. Rev. X 5, 041037 (2015).

[11] J.-M. Pirkkalainen, E. Damskägg, M. Brandt, F. Massel, and M. A. Sillanpää, Squeezing of Quantum Noise of Motion in a Micromechanical Resonator, Phys. Rev. Lett. 115, 243601 (2015).

[12] J. Klaers, Landauer's Erasure Principle in a Squeezed Thermal Memory, Phys. Rev. Lett. 122, 040602 (2019).

[13] F. DiFilippo, V. Natarajan, K. R. Boyce, and D. E. Pritchard, Classical Amplitude Squeezing for Precision Measurements, Phys. Rev. Lett. 68, 2859 (1992). 
[14] V. Natarajan, F. DiFilippo, and D. E. Pritchard, Classical Squeezing of an Oscillator for Subthermal Noise Operation, Phys. Rev. Lett. 74, 2855 (1995).

[15] A. Szorkovszky, G. A. Brawley, A. C. Doherty, and W. P. Bowen, Strong Thermomechanical Squeezing via Weak Measurement, Phys. Rev. Lett. 110, 184301 (2013).

[16] B. Yurke, P. G. Kaminsky, R. E. Miller, E. A. Whittaker, A. D. Smith, A. H. Silver, and R. W. Simon, Observation of 4.2-K Equilibrium-Noise Squeezing via a JosephsonParametric Amplifier, Phys. Rev. Lett. 60, 764 (1988).

[17] B. Yurke, L. R. Corruccini, P. G. Kaminsky, L. W. Rupp, A. D. Smith, A.H. Silver, R.W. Simon, and E. A. Whittaker, Observation of Parametric Amplification and Deamplification in a Josephson Parametric Amplifier, Phys. Rev. A 39, 2519 (1989).

[18] D. Rugar and P. Grütter, Mechanical Parametric Amplification and Thermomechanical Noise Squeezing, Phys. Rev. Lett. 67, 699 (1991).

[19] D. W. Carr, S. Evoy, L. Sekaric, H. G. Craighead, and J. M. Parpia, Parametric Amplification in a Torsional Microresonator, Appl. Phys. Lett. 77, 1545 (2000).

[20] J. Suh, M. D. LaHaye, P. M. Echternach, K. C. Schwab, and M. L. Roukes, Parametric Amplification and Back-Action Noise Squeezing by a Qubit-Coupled Nanoresonator, Nano Lett. 10, 3990 (2010).

[21] A. Vinante and P. Falferi, Feedback-Enhanced Parametric Squeezing of Mechanical Motion, Phys. Rev. Lett. 111, 207203 (2013).

[22] M. Poot, K. Y. Fong, and H. X. Tang, Classical NonGaussian State Preparation through Squeezing in an Optoelectromechanical Resonator, Phys. Rev. A 90 , 063809 (2014).

[23] S. Sonar, V. Fedoseev, M. J. Weaver, F. Luna, E. Vlieg, H. van der Meer, D. Bouwmeester, and W. Löffler, Strong Thermomechanical Squeezing in a Far-Detuned Membrane-in-the-Middle System, Phys. Rev. A 98, 013804 (2018).

[24] I. Mahboob, H. Okamoto, K. Onomitsu, and H. Yamaguchi, Two-Mode Thermal-Noise Squeezing in an Electromechanical Resonator, Phys. Rev. Lett. 113, 167203 (2014).

[25] Y. S. Patil, S. Chakram, L. Chang, and M. Vengalattore, Thermomechanical Two-Mode Squeezing in an UltrahighQ Membrane Resonator, Phys. Rev. Lett. 115, 017202 (2015).

[26] A. Pontin, M. Bonaldi, A. Borrielli, L. Marconi, F. Marino, G. Pandraud, G. A. Prodi, P. M. Sarro, E. Serra, and F. Marin, Dynamical Two-Mode Squeezing of Thermal Fluctuations in a Cavity Optomechanical System, Phys. Rev. Lett. 116, 103601 (2016).

[27] M. Dykman, Fluctuating Nonlinear Oscillators: From Nanomechanics to Quantum Superconducting Circuits, 1st ed. (Oxford University Press, Oxford, 2012).

[28] E. Buks and B. Yurke, Mass Detection with a Nonlinear Nanomechanical Resonator, Phys. Rev. E 74, 046619 (2006).

[29] R. Almog, S. Zaitsev, O. Shtempluck, and E. Buks, Noise Squeezing in a Nanomechanical Duffing Resonator, Phys. Rev. Lett. 98, 078103 (2007).

[30] M. I. Dykman and M. A. Krivoglaz, Theory of Fluctuational Transitions between Stable States of a Non Linear Oscillator, Zh. Eksp. Teor. Fiz. 77, 60 (1979).
[31] P. D. Drummond and D.F. Walls, Quantum-Theory of Optical Bistability. 1: Non-Linear Polarizability Model, J. Phys. A 13, 725 (1980).

[32] M. I. Dykman, D. G. Luchinsky, R. Mannella, P. V. E. McClintock, N. D. Stein, and N. G. Stocks, Supernarrow Spectral Peaks and High-Frequency Stochastic Resonance in Systems with Coexisting Periodic Attractors, Phys. Rev. E 49, 1198 (1994).

[33] C. Stambaugh and H. B. Chan, Supernarrow Spectral Peaks near a Kinetic Phase Transition in a Driven Nonlinear Micromechanical Oscillator, Phys. Rev. Lett. 97, 110602 (2006).

[34] M. I. Dykman, M. Marthaler, and V. Peano, Quantum Heating of a Parametrically Modulated Oscillator: Spectral Signatures, Phys. Rev. A 83, 052115 (2011).

[35] M. I. Dykman, Periodically Modulated Quantum Nonlinear Oscillators, in Fluctuating Nonlinear Oscillators: From Nanomechanics to Quantum Superconducting Circuits, edited by M.I. Dykman (Oxford University Press, Oxford, 2012), pp. 165-197.

[36] S. André, L. Guo, V. Peano, M. Marthaler, and G. Schön, Emission Spectrum of the Driven Nonlinear Oscillator, Phys. Rev. A 85, 053825 (2012).

[37] K. Y. Fong, W. H. P. Pernice, and H. X. Tang, Frequency and Phase Noise of Ultrahigh $Q$ Silicon Nitride Nanomechanical Resonators, Phys. Rev. B 85, 161410(R) (2012).

[38] R. Bonifacio and L. A. Lugiato, Photon Statistics and Spectrum of Transmitted Light in Optical Bistability, Phys. Rev. Lett. 40, 1023 (1978).

[39] T. Faust, P. Krenn, S. Manus, J. P. Kotthaus, and E. M. Weig, Microwave Cavity-Enhanced Transduction for Plug and Play Nanomechanics at Room Temperature, Nat. Commun. 3, 728 (2012).

[40] Q. P. Unterreithmeier, T. Faust, and J. P. Kotthaus, Damping of Nanomechanical Resonators, Phys. Rev. Lett. 105, 027205 (2010).

[41] M. Gad-el Hak, The MEMS Handbook (CRC Press, Boca Raton, FL, 2002).

[42] S. S. Verbridge, J. M. Parpia, R. B. Reichenbach, L. M. Bellan, and H. G. Craighead, High Quality Factor Resonance at Room Temperature with Nanostrings under High Tensile Stress, J. Appl. Phys. 99, 124304 (2006).

[43] Q. P. Unterreithmeier, E. M. Weig, and J. P. Kotthaus, Universal Transduction Scheme for Nanomechanical Systems Based on Dielectric Forces, Nature (London) 458, 1001 (2009).

[44] J. Rieger, T. Faust, M. J. Seitner, J. P. Kotthaus, and E. M. Weig, Frequency and Q Factor Control of Nanomechanical Resonators, Appl. Phys. Lett. 101, 103110 (2012).

[45] See Supplemental Material at http://link.aps.org/ supplemental/10.1103/PhysRevX.10.021066 for the theoretical analysis of the thermal noise squeezing and its relation with the power spectrum in a driven Duffing resonator, as well as the spectral manifestation of squeezing in the quantum regime. Presented there are also the characterization of the nanostring resonator, the calibration of the detection setup, the analysis of the data, further supplementary experimental data, and a discussion of the impact of frequency fluctuations on the direct homodyne 
detection of the noise quadratures of a weakly damped nanomechanical resonator.

[46] L. D. Landau and E. M. Lifshitz, Theory of Elasticity, 3rd ed. (Butterworth-Heinemann, Oxford, 1986).

[47] A. H. Nayfeh and D. T. Mook, Nonlinear Oscillations, 7th ed. (Wiley, New York, 1995).

[48] M. I. Dykman and M. A. Krivoglaz, Theory of Nonlinear Oscillators Interacting with a Medium, in Sov. Phys. Reviews, Vol. 5, edited by I. M. Khalatnikov (Harwood Academic, New York, 1984), pp. 265-441, https://web.pa .msu.edu/people/dykman/pub06/DKreview84.pdf.

[49] M. I. Dykman and M. A. Krivoglaz, Classical Theory of Nonlinear Oscillators Interacting with a Medium, Phys. Status Solidi B 48, 497 (1971).

[50] V. I. Arnold, Mathematical Methods of Classical Mechanics (Springer, New York, 1989).

[51] T. Antoni, K. Makles, R. Braive, T. Briant, P.-F. Cohadon, I. Sagnes, I. Robert-Philip, and A. Heidmann, Nonlinear
Mechanics with Suspended Nanomembranes, Europhys. Lett. 100, 68005 (2012).

[52] V. Peano and M. Thorwart, Quasienergy Description of the Driven Jaynes-Cummings Model, Phys. Rev. B 82, 155129 (2010).

[53] J. S. Aldridge and A. N. Cleland, Noise-Enabled Precision Measurements of a Duffing Nanomechanical Resonator, Phys. Rev. Lett. 94, 156403 (2005).

[54] C. Stambaugh and H. B. Chan, Noise-Activated Switching in a Driven Nonlinear Micromechanical Oscillator, Phys. Rev. B 73, 172302 (2006).

[55] M. Defoort, V. Puller, O. Bourgeois, F. Pistolesi, and E. Collin, Scaling Laws for the Bifurcation Escape Rate in a Nanomechanical Resonator, Phys. Rev. E 92, 050903(R) (2015).

[56] Data and analysis code are available at https://doi.org/ 10.5281/zenodo.3821371. 